\title{
Belgeo
}

Revue belge de géographie

$1 \mid 2018$

Miscellaneous

\section{La géographie humaine et ses cursus au sein des universités belges et européennes}

Human geography and its curricula in Belgian and European universities

Jean-Marie Halleux et Christophe Breuer

\section{(2) OpenEdition}

\section{Journals}

Édition électronique

URL : http://journals.openedition.org/belgeo/20899

DOI : 10.4000/belgeo.20899

ISSN : 2294-9135

Éditeur :

National Committee of Geography of Belgium, Société Royale Belge de Géographie

\section{Référence électronique}

Jean-Marie Halleux et Christophe Breuer, «La géographie humaine et ses cursus au sein des universités belges et européennes », Belgeo [En ligne], 1 | 2018, mis en ligne le 10 avril 2018, consulté le 15 janvier 2020. URL : http://journals.openedition.org/belgeo/20899; DOI : 10.4000/belgeo.20899

Ce document a été généré automatiquement le 15 janvier 2020.

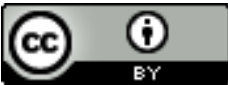

Belgeo est mis à disposition selon les termes de la licence Creative Commons Attribution 4.0 International. 


\section{La géographie humaine et ses cursus au sein des universités belges et européennes}

Human geography and its curricula in Belgian and European universities

Jean-Marie Halleux et Christophe Breuer

\section{Introduction}

1 Les universités européennes contribuent à un double mouvement. D'un côté, elles participent à la consolidation d'un espace européen de l'enseignement universitaire qui se traduit par la reconnaissance mutuelle de cycles d'études harmonisés (bachelier, master, doctorat). D'un autre côté, elles diversifient et spécialisent leurs formations afin de répondre à une demande croissante de professionnalisation des cursus et à l'évolution des attentes sociétales vis-à-vis des universités. Dans ce contexte, certaines disciplines universitaires sont mises sous tension, écartelées entre un enseignement disciplinaire «traditionnel » et des formations davantage "professionnalisantes » construites par et autour de communautés d'enseignants-chercheurs de plus en plus spécialisées. La géographie n'échappe pas à cette mise sous tension. Au contraire, elle y est particulièrement soumise par sa position ambivalente, à l'interface des sciences naturelles et des sciences humaines (Viles, 2005, Breuer, 2009).

2 La recherche synthétisée dans cet article porte sur l'enseignement universitaire de la géographie humaine. Face aux constats que nous venons de rappeler, nous avons analysé la situation de ce domaine au sein de six pays européens : la Belgique, la France, les Pays-Bas, l'Allemagne, le Royaume-Uni et la Suisse. Ces analyses ont été menées afin d'apporter des éléments de réponse aux questions suivantes :

- comment s'organise l'enseignement de la géographie universitaire dans les six pays analysés?

- quelle y est la place occupée par la géographie humaine dans l'enseignement universitaire de la géographie? 
- quelles y sont les relations entre la géographie humaine, la géographie physique et la géomatique?

- quelles y sont les relations entre la géographie humaine et les sciences connexes?

- comment la géographie humaine y fait-elle face à la problématique de la professionnalisation?

3 Afin de répondre à ces questions, nous avons comparé des programmes d'enseignement en nous focalisant sur les formations de premier cycle. Nous avons choisi cette approche car elle représente un point de départ approprié pour rendre compte de l'organisation générale des universités en matière de géographie humaine. En l'absence de base de données européennes des formations universitaires, cette analyse ne peut être exhaustive. Pour autant, il nous semble que, pour les six pays retenus, les éléments les plus généraux et les plus représentatifs ont pu être mis en évidence.

4 À l'issue de cette introduction, l'article se poursuit par une première section qui vise à préciser le champ couvert par le domaine de la géographie humaine. La deuxième section est ensuite dédiée aux programmes de bachelier dispensés au sein des universités belges. Après cette section sur les formations belges, nous nous intéressons aux formations étrangères, ce qui nous conduira à discerner trois modèles distincts d'enseignement de la géographie. Nous terminons ensuite par une discussion où nous constatons que le modèle pratiqué en Belgique n'est guère favorable à l'épanouissement de la géographie humaine.

\section{Le domaine de la géographie humaine au sein de la géographie}

5 Notre travail ne vise pas à développer une réflexion épistémologique sur la géographie en général ou sur la géographie humaine en particulier. Pour autant, il nous semble impossible de faire l'économie d'une présentation du champ couvert par ce domaine si nous voulons rencontrer nos principaux objectifs. Pour ce faire, nous développons cidessous les trois points suivants : la question de la définition des disciplines, la question du positionnement variable de la géographie au sein des universités et la question de l'employabilité des diplômés.

\section{Quelques définitions de base}

6 La géographie peut être définie comme la discipline scientifique qui étudie comment l'espace influence et interagit avec les processus sociaux et biophysiques :

[...] no definition of geography will satisfy everyone, and nor should it. But one possible definition of the contemporary discipline is: (the study of) the ways in which space is involved in the operation and outcome of social and biophysical processes (Gregory, 2009, p. 288).

La géographie est donc à la fois une science naturelle et une science humaine, ce qui tranche avec le découpage classique des disciplines scientifiques articulé par la scission entre sciences naturelles et sciences humaines. Cette situation explique pourquoi, comme nous le détaillons ci-dessous, la géographie se caractérise par un positionnement variable au sein des structures universitaires, avec des appartenances facultaires relevant tantôt des sciences humaines, tantôt des sciences naturelles (Hall et al., 2015). 
8 Le fait que la géographie soit une discipline à la croisée des sciences humaines et des sciences naturelles explique aussi pourquoi elle entretient d'étroites relations avec de nombreuses disciplines connexes. Le modèle classique d'André Cailleux (1973, p. 490) permet de structurer ces relations à partir de quatre subdivisions de la géographie : la géographie mathématique (que nous appellerions aujourd'hui la géomatique), la géographie physique, la biogéographie et la géographie humaine. Pour la géographie physique et pour la géographie mathématique, les relations les plus étroites sont respectivement tissées avec, d'un côté, la géologie, la météorologie ou l'hydrographie et, de l'autre côté, l'astronomie, la géométrie et la photogrammétrie. S'agissant de la géographie humaine, les liens privilégiés se rapportent aux sciences humaines et, en particulier, à l'économie, à l'histoire, à la sociologie et à la démographie.

9 Dans un ouvrage de référence sur les concepts fondamentaux de la géographie humaine, Antoine Bailly en formule une définition classique à laquelle nous n'avons rien à ajouter :

Nombre de géographes contemporains définissent la géographie humaine par trois questions «qui », «quoi », « où ». La première interrogation concerne les groupes qui occupent l'espace avec leurs valeurs, leurs genres de vie et leur vécu. La deuxième a trait aux productions économiques, sociales et culturelles de ces groupes grâce aux ressources, aux organisations, aux technologies et aux échanges. Quant à la troisième, la plus spécifique en géographie, elle aborde le problème des localisations, de la structuration des distributions spatiales et du sens des lieux. Cette dernière question nous renvoie aux pratiques spatiales, à la connaissance que l'homme a de son lieu de vie et à l'utilisation qu'il en fait. Ces trois questions doivent être complétées, pour que la géographie humaine soit vraiment globale, par le "pourquoi ", le "comment " et le "jusqu'où ", mettant ainsi en lumière les objectifs des individus et des groupes, les processus dans l'établissement des relations de pouvoirs dans l'espace, et les limites, seuils et discontinuités des pratiques humaines. Par ces questions, la géographie humaine se place délibérément dans le courant des sciences sociales, car toute étude géographique pose avant tout des questions privilégiant l'homme, ses représentations et ses pratiques (Bailly, 1995, pp. 18-19).

\section{Le positionnement variable de la géographie au sein des universités}

Le tableau 1 porte sur vingt universités particulièrement reconnues pour leur enseignement de la géographie. Il a été établi sur la base du travail mené par Leng et ses collègues (2017) et il illustre le fait que géographie se caractérise par un positionnement variable au sein des structures universitaires.

11 Il ressort du tableau 1 que les termes «géographie» ou "géographiques » peuvent apparaître au premier niveau de l'organisation hiérarchisée des universités, c'est-à-dire le niveau qui s'apparente classiquement à la notion de faculté. Par exemple, nous trouvons la mention du terme "géographie" au premier niveau des universités d'Oxford et de Cambridge. Un second élément qui ressort du tableau 1 correspond à l'hétérogénéité des premiers niveaux auxquels la géographie appartient. À l'instar de la situation belge, ce premier niveau peut correspondre aux sciences. Sur la base de l'échantillon analysé, il s'agit là d'une configuration finalement assez minoritaire. Pour les universités allemandes, nous trouvons principalement une appartenance au domaine des "géosciences». S'agissant du cas américain, nous trouvons, d'une part, des appartenances au domaine des sciences humaines et, d'autre part, des appartenances à de vastes structures facultaires en "Arts and Sciences» qui assurent 
tant des enseignements en sciences exactes (mathématiques, chimie, physique...) qu'en sciences sociales (psychologie, sociologie, anthropologie, histoire...).

La situation au sein des universités françaises n'a pas été prise en compte par les analyses de Leng et al. (2017). Afin de combler cette lacune, nous nous sommes appuyés sur une liste des UFR (Unité de formation et de recherche) qui assurent des enseignements de géographie au sein de l'enseignement supérieur français ${ }^{1}$. Il ressort de cette liste que la géographie est enseignée au sein de 57 UFR et que les termes " géographie ", "géographiques" ou "territoires » apparaissent 15 fois au premier niveau de l'organisation hiérarchisée des institutions concernées. Il ressort aussi de l'analyse du cas français que la géographie est très fréquemment couplée au domaine de l'aménagement du territoire et de l'urbanisme. Cette association peut concerner tant le premier que le second niveau hiérarchique. Par exemple, l'Universités Rennes 2 compte un département «Géographie et aménagement de l'espace » (niveau 2) au sein de son UFR « Sciences sociales » (niveau 1).

13 Concernant les domaines de premier rang auxquels la géographie appartient, trois possibilités existent en France. La première correspond à une référence explicite à la géographie dès le premier rang hiérarchique. La deuxième possibilité est l'appartenance à un premier rang qui relève de l'aménagement et de l'urbanisme, à l'image de l'Institut d'urbanisme de Lyon. La troisième possibilité, de loin la plus importante, est une appartenance au domaine général des sciences sociales, avec des appartenances du type «UFR des lettres et sciences sociales » ou « Faculté des lettres et des sciences humaines ». Cette situation tranche vis-à-vis du contexte belge avec l'appartenance institutionnelle des départements de géographie aux facultés des sciences. 
Tableau 1. Le positionnement de la géographie dans l'organisation des universités.

\begin{tabular}{|c|c|c|c|c|}
\hline Pays & Université & Dénomination du niveau 1 & Dénomination du niveau 2 & $\begin{array}{l}\text { Domaine } \\
\text { du rang } 1\end{array}$ \\
\hline \multirow{6}{*}{$\begin{array}{l}\text { Royaume- } \\
\text { Uni }\end{array}$} & $\begin{array}{l}\text { University of } \\
\text { Oxford }\end{array}$ & $\begin{array}{l}\text { Faculty Board of Anthropology } \\
\text { and Geography }\end{array}$ & $\begin{array}{l}\text { School of Geography and } \\
\text { the Environment }\end{array}$ & $\begin{array}{l}\text { Sciences } \\
\text { humaines }\end{array}$ \\
\hline & $\begin{array}{l}\text { University of } \\
\text { Leeds }\end{array}$ & Faculty of Environment & School of Geography & $\begin{array}{l}\text { Sciences de } \\
\text { l'environnement }\end{array}$ \\
\hline & $\begin{array}{l}\text { University of } \\
\text { Cambridge }\end{array}$ & $\begin{array}{l}\text { Faculty of Earth Science and } \\
\text { Geography }\end{array}$ & Department of Geography & Géosciences \\
\hline & $\begin{array}{l}\text { Durham } \\
\text { University }\end{array}$ & Department of Geography & & Indéterminé \\
\hline & Bristol University & Faculty of Science & $\begin{array}{l}\text { School of Geographical } \\
\text { Sciences }\end{array}$ & Sciences \\
\hline & $\begin{array}{l}\text { University of } \\
\text { Exeter }\end{array}$ & $\begin{array}{l}\text { College of Life and } \\
\text { Environmental Sciences }\end{array}$ & Geography & $\begin{array}{l}\text { Sciences de } \\
\text { l'environnement }\end{array}$ \\
\hline \multirow{4}{*}{ Chine } & $\begin{array}{l}\text { Beijing Normal } \\
\text { University }\end{array}$ & School of Geography & $\begin{array}{l}\text { Department of } \\
\text { Geographical Sciences }\end{array}$ & Indéterminé \\
\hline & Peking University & $\begin{array}{l}\text { College of Urban and } \\
\text { Environmental Sciences }\end{array}$ & $\begin{array}{l}\text { Department of Research } \\
\text { and Environmental } \\
\text { Geography } \\
\text { Department of Physical } \\
\text { Geography } \\
\text { Department of Urban and } \\
\text { Economical Geography }\end{array}$ & $\begin{array}{l}\text { Sciences de } \\
\text { l'environnement }\end{array}$ \\
\hline & \multirow{2}{*}{$\begin{array}{l}\text { East China } \\
\text { Normal University }\end{array}$} & School of Geographic Science & $\begin{array}{l}\text { Geographic Science } \\
\text { Geographical Information } \\
\text { Science }\end{array}$ & Indéterminé \\
\hline & & $\begin{array}{l}\begin{array}{l}\text { School of Urban and Regional } \\
\text { Science }\end{array} \\
\end{array}$ & $\begin{array}{l}\text { Department of Human } \\
\text { Geography }\end{array}$ & $\begin{array}{l}\text { Sciences } \\
\text { humaines } \\
\end{array}$ \\
\hline \multirow{6}{*}{ Allemagne } & $\begin{array}{l}\text { University of } \\
\text { Cologne }\end{array}$ & Department of Geoscience & Geography Institute & Géosciences \\
\hline & $\begin{array}{l}\text { Meidelberg } \\
\text { University }\end{array}$ & $\begin{array}{l}\text { Department of Chemistry and } \\
\text { Geoscience }\end{array}$ & Institute of Geography & Sciences \\
\hline & $\begin{array}{l}\text { University of } \\
\text { Gottigen }\end{array}$ & $\begin{array}{l}\text { Faculty of Geosciences and } \\
\text { Geography }\end{array}$ & Institute of Geography & Géosciences \\
\hline & $\begin{array}{l}\text { University of } \\
\text { Freiburg }\end{array}$ & $\begin{array}{l}\text { Faculty of Environment and } \\
\text { Natural Resources }\end{array}$ & Institute of Geography & $\begin{array}{l}\text { Sciences de } \\
\text { leenvironnement }\end{array}$ \\
\hline & $\begin{array}{l}\text { University of } \\
\text { Bonn }\end{array}$ & Department of Geoscience & Institute of Geography & Géosciences \\
\hline & $\begin{array}{l}\text { Humboldt } \\
\text { University of } \\
\text { Berlin }\end{array}$ & $\begin{array}{l}\text { Faculty of Mathematics and } \\
\text { Natural Sciences II }\end{array}$ & Geography Department & Sciences \\
\hline \multirow{5}{*}{ États-Unis } & Boston University & College of Arts and Sciences & $\begin{array}{l}\text { Department of Earth and } \\
\text { Environment }\end{array}$ & $\begin{array}{l}\text { "Arts and } \\
\text { Sciences" }\end{array}$ \\
\hline & $\begin{array}{l}\text { University of } \\
\text { Maryland College } \\
\text { Park UM }\end{array}$ & $\begin{array}{l}\text { College of Behavioural and } \\
\text { Social sciences }\end{array}$ & $\begin{array}{l}\text { Department of } \\
\text { Geographical Sciences }\end{array}$ & $\begin{array}{l}\text { Sciences } \\
\text { humaines }\end{array}$ \\
\hline & $\begin{array}{l}\text { University of } \\
\text { Colorado Bulder }\end{array}$ & College of Arts and Sciences & Department of Geography & $\begin{array}{l}\text { "Arts and } \\
\text { Sciences" }\end{array}$ \\
\hline & $\begin{array}{l}\text { University of } \\
\text { California Santa } \\
\text { Barbara } \\
\end{array}$ & College of Letters and Sciences & Department of Geography & $\begin{array}{l}\text { "Arrs and } \\
\text { Sciences" }\end{array}$ \\
\hline & $\begin{array}{l}\text { University of } \\
\text { Calfornia Los } \\
\text { Angeles }\end{array}$ & Social Sciences Division & Department of Geography & $\begin{array}{l}\text { Sciences } \\
\text { humaines }\end{array}$ \\
\hline
\end{tabular}

Note méthodologique : les cases grisées font référence à des universités où les appellations « géographie » ou « géographiques » apparaissent au niveau 1 de l'organisation des structures universitaires.

Source : S. Leng et al., 2017, p. 12.

\section{L'employabilité des diplômés en géographie}

14 Comme l'ont résumé Arrowsmith et ses collègues, l'employabilité des diplômés en géographie s'est fortement renforcée et diversifiée durant les dernières décennies :

[...] there is a strong demand in many countries for graduates with geographic perspectives and technological competencies, with commensurate growth in geographical and geospatial career fields (Arrowsmith et al., 2011, p. 365). Les enquêtes post-master démontrent la diversité des métiers exercés par des géographes, avec toutefois une prédominance des domaines du développement territorial, de la gestion de l'information spatiale, de la pédagogie et de l'environnement ${ }^{2}$.

16 Le développement de l'employabilité des diplômés en géographie résulte de plusieurs facteurs. Le premier tient aux besoins sociétaux croissants pour l'aménagement, le développement et l'environnement, trois domaines pour lesquels l'expertise des géographes est particulièrement recherchée.

17 Le développement de l'employabilité résulte aussi de la professionnalisation des filières de formation (Arrowsmith et al., 2011; Whalley et al., 2011). La tendance vers la professionnalisation correspond à une évolution générale des systèmes éducatifs, de plus en plus soucieux des débouchés professionnels de leurs étudiants. En géographie comme ailleurs, il en a résulté le développement de la pédagogie par projet, la mise aux 
programmes de stages professionnels et une attention croissante aux soft skills et à la maîtrise des langues étrangères.

Une autre explication majeure du renforcement de l'employabilité des diplômés en géographie tient au développement des techniques de la géomatique, des systèmes d'informations géographiques (SIG) et du big data. Cette évolution explique l'important développement que connait aujourd'hui la géographie universitaire aux États-Unis (Murphy, 2007).

\section{Les programmes de bachelier en Belgique}

\section{Méthodologie}

19 Notre méthodologie est basée sur l'analyse des programmes de bachelier au sein des sept institutions universitaires belges qui organisent un premier cycle en géographie. Il s'agit des institutions suivantes: la Katholieke Universiteit Leuven (KU Leuven), l'Universiteit Gent (UGent), la Vrije Universiteit Brussel (VUB), l'Université catholique de Louvain (UCL), l'Université libre de Bruxelles (ULB), l'Université de Liège (ULiège) et l'Université de Namur (UNamur). Ces universités appartiennent à deux rôles linguistiques relativement cloisonnés sur le plan institutionnel: les trois premières universités relèvent de la Communauté flamande (rôle néerlandophone) et les quatre autres de la Communauté française de Belgique (rôle francophone).

Les données rassemblées dans le tableau 2 ont été recensées en septembre et en octobre 2016, à partir des sites internet des différentes institutions. Les crédits de cours (ECTS $\left.{ }^{3}\right)$ de chaque formation y sont ventilés en quatre rubriques:

- "sciences naturelles et sciences mathématiques ", qui intègre les enseignements de chimie, physique, mathématique (y compris les cours de statistiques) et de biologie (y compris les cours d'écologie) ;

- "géographie humaine et sciences humaines » qui, en plus des enseignements de géographie humaine, intègre les cours d'économie, de sociologie, d'histoire et également de philosophie ;

- "géographie physique et géosciences ", qui intègre les cours de géologie ;

- " géomatique et informatique »;

- "autres", qui rassemble les cours de sciences religieuses et de communications scientifiques et al. 
Tableau 2. Crédits par types de cours pour les formations de bachelier en géographie au sein des universités belges - Calculs établis sur la base d'une maximisation des cours en géographie humaine et sciences humaines.

\begin{tabular}{|c|c|c|c|c|c|c|c|}
\hline Université & $\begin{array}{c}\text { Sciences } \\
\text { naturelles } \\
\text { et mathé- } \\
\text { matiques. }\end{array}$ & $\begin{array}{c}\text { Géo. } \\
\text { humaine et } \\
\text { sc. } \\
\text { humaines }\end{array}$ & $\begin{array}{c}\text { Géo. } \\
\text { physique et } \\
\text { géosciences }\end{array}$ & $\begin{array}{c}\text { Géomatique } \\
\text { et } \\
\text { informatique }\end{array}$ & Langues & Autres & TOTAL \\
\hline KULeuven & $\begin{array}{c}35 \\
19,4 \% \\
\end{array}$ & $\begin{array}{c}73 \\
40,6 \% \\
\end{array}$ & $\begin{array}{c}39 \\
21,7 \% \\
\end{array}$ & $\begin{array}{c}27 \\
15,0 \% \\
\end{array}$ & $\begin{array}{c}0 \\
0 \% \\
0 \%\end{array}$ & $\begin{array}{c}6 \\
3,3 \% \\
\end{array}$ & $\begin{array}{c}180 \\
100 \% \\
\end{array}$ \\
\hline UCL & $\begin{array}{c}65 \\
35,7 \%\end{array}$ & $\begin{array}{c}43 \\
23,6 \%\end{array}$ & $\begin{array}{c}38 \\
20,9 \%\end{array}$ & $\begin{array}{c}24 \\
13,2 \%\end{array}$ & $\begin{array}{c}9 \\
4,9 \%\end{array}$ & $\begin{array}{c}3 \\
1,6 \%\end{array}$ & $\begin{array}{c}182 \\
100 \%\end{array}$ \\
\hline UGent & $\begin{array}{c}39 \\
21,7 \% \\
\end{array}$ & $\begin{array}{c}59,5 \\
33,1 \%\end{array}$ & $\begin{array}{c}40,5 \\
22,5 \%\end{array}$ & $\begin{array}{c}41 \\
22,8 \%\end{array}$ & $\begin{array}{c}0 \\
0 \%\end{array}$ & $\begin{array}{c}0 \\
0 \%\end{array}$ & $\begin{array}{c}180 \\
100 \%\end{array}$ \\
\hline ULB & $\begin{array}{c}55 \\
30,6 \%\end{array}$ & $\begin{array}{c}62,5 \\
34,7 \%\end{array}$ & $\begin{array}{c}32,5 \\
18,1 \%\end{array}$ & $\begin{array}{c}20 \\
11,1 \%\end{array}$ & $\begin{array}{c}10 \\
5,6 \%\end{array}$ & $\begin{array}{c}0 \\
0 \%\end{array}$ & $\begin{array}{c}180 \\
100 \%\end{array}$ \\
\hline ULiège & $\begin{array}{c}55 \\
30,6 \% \\
\end{array}$ & $\begin{array}{c}39 \\
21,7 \% \\
\end{array}$ & $\begin{array}{c}37,5 \\
20,8 \% \\
\end{array}$ & $\begin{array}{c}38,5 \\
21,4 \% \\
\end{array}$ & $\begin{array}{c}10 \\
5,6 \% \\
\end{array}$ & $\begin{array}{c}0 \\
0 \%\end{array}$ & $\begin{array}{c}180 \\
100 \%\end{array}$ \\
\hline UNamur & $\begin{array}{c}49 \\
27,2 \%\end{array}$ & $\begin{array}{c}57 \\
31,7 \%\end{array}$ & $\begin{array}{c}31,5 \\
17,5 \%\end{array}$ & $\begin{array}{c}31,5 \\
17,5 \%\end{array}$ & $\begin{array}{c}9 \\
5,0 \%\end{array}$ & $\begin{array}{c}2 \\
1,1 \%\end{array}$ & $\begin{array}{c}180 \\
100 \%\end{array}$ \\
\hline VUB & $\begin{array}{c}42 \\
23,3 \%\end{array}$ & $\begin{array}{c}80 \\
44,4 \%\end{array}$ & $\begin{array}{c}34 \\
18,9 \%\end{array}$ & $\begin{array}{c}24 \\
13,3 \%\end{array}$ & $\begin{array}{c}0 \\
0 \%\end{array}$ & $\begin{array}{c}0 \\
0 \%\end{array}$ & $\begin{array}{c}180 \\
100 \%\end{array}$ \\
\hline
\end{tabular}

Source : sites internet des universités (septembre et octobre 2016)

21 Pour les différents programmes, la part effective des enseignements dans chacune des rubriques considérées dépend fortement des choix posés par les étudiants. Cette situation peut fortement complexifier l'analyse des programmes de cours et elle trouve son origine dans les choix d'options, dans l'orientation donnée aux travaux intégrés et aux travaux de fin d'étude ou, lorsque ce choix existe, par la sélection d'orientations mineures ${ }^{4}$. Face à cette situation, nous avons sélectionné une méthode de calcul basée sur des choix d'étudiants qui se feraient systématiquement par la sélection d'enseignements appartenant aux domaines de la géographie humaine et des sciences humaines. Par exemple, s'agissant de l'Université de Liège, nous avons considéré le scénario où l'étudiant de troisième année réalise son travail de fin de bachelier - un enseignement qui représente 5 crédits - dans le domaine de la géographie humaine.

Sur la base de leur intitulé, le rattachement des cours et des crédits afférents aux rubriques considérées ne pose en général pas de problème particulier. En cas de doute, les descriptifs des cours ont été consultés afin de les affecter à la rubrique correcte. Dans certains cas, la consultation des engagements pédagogiques n'a pas permis de trancher de manière indiscutable. Cela concerne en particulier les cours de géographie dédiés à des exercices intégrés, à des travaux de terrain ou à des matières telles que le paysage ou la géographie régionale. Pour ces situations, nous avons divisé les ECTS correspondant en les allouant de manière égale aux matières potentiellement concernées. Par exemple, certains cours dédiés à la géographie régionale ou à l'analyse paysagère ont été attribués pour une moitié à la géographie humaine et pour l'autre moitié à la géographie physique.

\section{Analyse}

La première observation à retirer du tableau 2 se rapporte au poids important des cours de la rubrique "sciences naturelles et sciences mathématiques ». Cette situation est en réalité une spécificité belge, que nous n'avons retrouvée nulle part ailleurs dans les programmes analysés. Elle peut en partie s'expliquer par le fait que les études de géographie soient organisées par les facultés de sciences. À propos des enseignements 
en "sciences naturelles et sciences mathématiques », l'analyse témoigne de différences notables de part et d'autre de la frontière linguistique, avec un nombre plus important de crédits en Belgique francophone (entre 49 et 65 crédits) qu'en Belgique néerlandophone (entre 35 et 42 crédits). Une seconde différence notable entre les universités des deux régimes linguistiques se rapporte aux cours de langues étrangères (anglais), qui sont obligatoires dans les cursus francophones, mais absents du programme ou uniquement disponibles en option pour les cursus néerlandophones.

En comparaison des autres universités, le poids des cours dédiés à la géomatique et à l'informatique est plus élevé à l'ULiège et à l'UGent. Ce résultat n'est guère surprenant puisque ces deux institutions sont les seules à organiser un master de géographie à orientation géomatique-géométrologie. Pour les cours de géographie physique et de géosciences, aucune tendance nette ne se dégage. Les cours obligatoires qui appartiennent à ces domaines représentent approximativement $20 \%$ des crédits au sein des sept universités considérées.

Concernant le poids de la géographie humaine et des sciences humaines, le jeu des options dont rend compte le tableau 2 conduit à des disparités assez sensibles, depuis 39 crédits $(21,7 \%)$ à l'ULiège jusqu'à 80 crédits $(44,4 \%)$ à la VUB.

\section{Les programmes de bachelier à l'étranger}

Comme nous allons le détailler, notre analyse internationale des formations de bachelier en géographie atteste d'une très grande diversité. À ce jour, les cursus demeurent très marqués par des histoires nationales divergentes et la dynamique de convergence initiée par le processus européen de Bologne ne semble avoir eu qu'un impact limité sur l'enseignement universitaire de la géographie. Du point de vue méthodologique, cette extrême diversité des programmes rend impossible l'utilisation d'une grille de lecture unique de ceux-ci. Pour autant, cette impossibilité d'établir des calculs de crédits parfaitement homogènes ne nous a pas empêchés de mettre en exergue la coexistence de modèles distincts.

\section{La France}

L'exercice de dépouillement détaillé des programmes de premier cycle a été réalisé pour trois universités françaises : l'Université de Rennes 2, l'Université Paris-Sorbonne (Paris 4) et l'Université de Strasbourg. Nous nous limitons ici à ces trois institutions car la consultation des programmes de cinq autres universités ne témoigne pas de différences notables ${ }^{5}$.

En France, la formation de premier cycle est dénommée "licence». Les licences françaises du domaine de la géographie différencient en général deux parcours: un parcours "géographie au sens strict» et un parcours "géographie-aménagement ». Dans certaines institutions, il existe également un troisième parcours en "géographieenvironnement». Cette variété dans les programmes témoigne de l'évolution opérée depuis les années quatre-vingt afin de renforcer l'employabilité des diplômés. Cette évolution, dont nous avons vu ci-dessus qu'elle est générale aux systèmes de formation, a été bien résumée par B. Dézert et J. Bastié pour les cursus français en géographie. Les départements de géographie des universités françaises 
[...] ont tourné leurs enseignements et formations, en plus de la formation des futurs professeurs et chercheurs en géographie, vers des filières professionnelles pluridisciplinaires, comme l'aménagement du territoire et des villes, les questions de défense de l'environnement naturel, les systèmes d'information géographique et la science régionale. Les géographes sont de plus en plus formés pour être des conseillers des décideurs politiques et économiques régionaux, des experts en logistique dans les entreprises qui exportent hors d'Europe (Dézert et Bastié, 2004, p. 5 de la version en ligne).

Le tableau 3 présente une comptabilité des crédits comparable à celle utilisée pour établir le tableau 2. Vu les importantes différences entre les programmes belges et français, nous avons toutefois modifié les rubriques considérées, en faisant mention de deux rubriques spécifiques pour les cours des domaines de l'aménagement et de l'environnement. Par ailleurs, nous n'avons pas comptabilisé les crédits des options sur la base d'une maximisation des crédits en " géographie humaine et sciences humaines " car, parmi les programmes analysés, les étudiants qui le souhaitent peuvent généralement choisir des options qui ne comportent que des enseignements de géographie humaine ou de sciences humaines. En plus des cours liés à la géographie humaine ou aux sciences humaines, les options disponibles au sein des programmes analysés correspondent généralement à des cours de géographie physique ou à des cours destinés aux étudiants qui se préparent à une carrière d'enseignant.

Tableau 3. Crédits par types de cours pour les formations de bachelier en géographie au sein d'un échantillon d'universités françaises.

\begin{tabular}{|l|c|c|c|c|c|c|c|}
\hline $\begin{array}{l}\text { Université et } \\
\text { filière }\end{array}$ & $\begin{array}{c}\text { Sciences } \\
\text { naturelles } \\
\text { et mathé- } \\
\text { matiques }\end{array}$ & $\begin{array}{c}\text { Géo. } \\
\text { humaine et } \\
\text { sc. } \\
\text { humaines }\end{array}$ & $\begin{array}{c}\text { Aména- } \\
\text { gement }\end{array}$ & $\begin{array}{c}\text { Géo. } \\
\text { physique et } \\
\text { géosciences }\end{array}$ & $\begin{array}{c}\text { Géoma- } \\
\text { tique et } \\
\text { informa- } \\
\text { tique }\end{array}$ & $\begin{array}{c}\text { Environ- } \\
\text { nement }\end{array}$ & $\begin{array}{c}\text { Langues } \\
\text { et options }\end{array}$ \\
\hline $\begin{array}{l}\text { Rennes2 } \\
\text { Géographie }\end{array}$ & 10 & 87 & 8,5 & 18 & 27 & 9,5 & 20 \\
\hline Rennes2 & $5,6 \%$ & $48,3 \%$ & $4,7 \%$ & $10,0 \%$ & $15,0 \%$ & $5,3 \%$ & $11,1 \%$ \\
Aménagement & $7,9 \%$ & $26,7 \%$ & $37,2 \%$ & $6,7 \%$ & $11,1 \%$ & $3,3 \%$ & $11,1 \%$ \\
\hline Rennes2 & 7 & 37,5 & 8,5 & 19 & 25,5 & 62,5 & 20 \\
Environnement & $3,9 \%$ & $20,8 \%$ & $4,7 \%$ & $10,6 \%$ & $14,2 \%$ & $34,7 \%$ & $11,1 \%$ \\
\hline Strasbourg & 15 & 39 & 0 & 33 & 21 & 0 & 69 \\
Géographie & $8,3 \%$ & $21,7 \%$ & $0 \%$ & $18,3 \%$ & $11,7 \%$ & $0 \%$ & $38,3 \%$ \\
\hline $\begin{array}{l}\text { Strasbourg } \\
\text { Aménagement }\end{array}$ & 12 & 43,5 & 30 & 30 & 18,5 & 3 & 40 \\
\hline Paris 4 & 15 & $24,2 \%$ & $16,7 \%$ & $16,7 \%$ & $10,3 \%$ & $1,7 \%$ & $22,2 \%$ \\
Géographie & $8,3 \%$ & $31,7 \%$ & $2,2 \%$ & $13,3 \%$ & $11,1 \%$ & $6,7 \%$ & $26,7 \%$ \\
\hline Paris 4 & 8 & 50 & 39 & 27 & 17 & 4 & 35 \\
Aménagement & $4,4 \%$ & $27,8 \%$ & $21,7 \%$ & $15,0 \%$ & $9,4 \%$ & $2,2 \%$ & $19,4 \%$ \\
\hline
\end{tabular}

Source : sites internet des universités (septembre et octobre 2016)

Pour les programmes consultés, les cours de sciences naturelles et de mathématiques se limitent principalement à des enseignements de statistiques, qui représentent entre 7 et 15 crédits. L'absence de sciences naturelles connexes dans les cursus français contraste avec la situation belge, où les enseignements en sciences chimiques, biologiques et physiques sont traditionnellement considérés comme des prérequis aux enseignements de la géographie en général et de la géographie physique en particulier.

31 En dépit de l'absence de cours dédiés spécifiquement à la chimie, à la physique ou à la biologie, les cursus français intègrent néanmoins une part non négligeable de cours de géographie physique (y compris de biogéographie) : jusqu'à 30 crédits par exemple à l'Université de Strasbourg en considérant tant la géomorphologie que la climatologie (auxquels il faut ajouter 3 crédits de géologie). 

des enseignements en sciences humaines autres que la géographie humaine. Sur les sept programmes pris en compte dans le tableau 3, les sciences humaines connexes (l'histoire, l'économie, la sociologie...) ne représentent que $7 \%$ de l'offre recensée dans la rubrique "géographie humaine et sciences humaines ". Conjuguée à l'absence des sciences physiques, chimiques et biologiques, cette faible représentation des sciences humaines connexes laisse à penser que les enseignants des cours de géographie se chargent directement de la mise à niveau des étudiants dans ces disciplines connexes, qu'il s'agisse de sciences naturelles ou de sciences humaines.

Concernant les cours de géomatique, les programmes relevés proposent entre 19 et 27 crédits. À l'exception de l'ULB, il s'agit là de volumes inférieurs à ce qui est proposé par les universités belges, tant en Belgique néerlandophone qu'en Wallonie. À propos des programmes français, mentionnons encore qu'ils intègrent une part non négligeable de langues : 11 crédits en moyenne dans les 7 programmes analysés.

\section{Les Pays-Bas}

Des bacheliers en géographie existent au sein de quatre universités néerlandaises: l'Université d'Amsterdam, l'Université d'Utrecht, l'Université Radboud de Nimègue et l'Université de Groningue (van der Vaart et al., 2004, p. 135). Les dénominations des quatre formations associent automatiquement le domaine de l'aménagement au domaine de la géographie, avec un «Bachelor Geografie, Planologie en Milieu » à Nimègue et des "Bachelors Sociale geografie en Planologie » à Amsterdam, Utrecht et Groningue. Dans ces différentes institutions, le bachelier en " géographie et aménagement » donne ensuite accès à des masters en géographie et à des masters en aménagement (planologie).

À l'exception de l'Université d'Utrecht, les sites internet des autres institutions ne permettent pas d'accéder aux programmes détaillés. Sur la base des informations disponibles, il en ressort une forte proximité avec les filières françaises quant aux poids respectifs de la géographie humaine, de la géographie physique, de la géomatique et des cours relevant du domaine de l'aménagement. En parallèle, selon les informations disponibles, nous ne trouvons pas trace de cours spécifiquement dédiés aux disciplines connexes à la géographie, qu'elles relèvent des sciences humaines (économie, sociologie, sciences politiques...) ou des sciences exactes (physique, chimie...).

À la différence du cas français, la géographie néerlandaise se caractérise par une scission institutionnelle entre la géographie humaine et la géographie physique. Par exemple, au sein de l'Université d'Utrecht, nous trouvons, d'un côté, la formation de " Bachelor Sociale geografie en Planologie » organisé par le «Departement of geography and spatial planning " et, d'un autre côté, une formation en "Earth Sciences " organisée conjointement par le «Departement of physical geography " et le "Department of Earth Sciences». Sur le plan institutionnel, les deux départements de géographie sont toutefois intégrés au sein de la même faculté (Faculty of Geosciences).

\section{L'Allemagne}

L'exercice de dépouillement des programmes de bachelier a été effectué pour six universités allemandes: l'Université Humboldt à Berlin, la Freie Universität Berlin, 
l'Université de Göttingen, l'Université de Heidelberg, l'Université de Bonn et l'Université de Cologne. Nous en avons synthétisé les résultats au sein du tableau 4. L'allocation des crédits au sein des universités allemandes n'étant pas explicite, ni pour les choix d'options ni pour l'organisation du travail de fin d'études (TFE), le tableau 4 intègre des colonnes pour ces catégories d'enseignement. De ce point de vue, il n'est donc pas directement comparable aux tableaux préparés pour les autres pays.

L'analyse des programmes allemands met en avant la coexistence de deux types de diplômes : des B.A. (Bachelor of Arts) et des B.Sc. (Bachelor of Science). L'analyse du cas allemand met aussi en avant la possibilité d'organiser des programmes où la géographie est choisie comme branche mineure. Cette possibilité existe par exemple à l'Université de Bonn, dans le cadre de B.A. en histoire, philosophie ou politique et société.

En comparaison de la France, nous trouvons dans les B.Sc. allemands une représentation plus forte des enseignements obligatoires en géographie physique et en géosciences. Pour ces matières, les programmes recensés correspondent à une moyenne de 39 crédits en Allemagne et de 23 crédits en France. Ce décalage est à lier aux organisations institutionnelles puisque, comme nous l'avons vu ci-dessus, les UFR et départements de géographie français appartiennent à des facultés de sciences sociales alors que les instituts de géographie allemands sont rattachés à des facultés scientifiques et, en particulier, à des facultés de géosciences qui organisent également des B.Sc. distincts en géosciences.

Tableau 4. Crédits par types de cours pour les formations de bachelier en géographie au sein d'un échantillon d'universités allemandes.

\begin{tabular}{|c|c|c|c|c|c|c|c|}
\hline $\begin{array}{l}\text { Université et } \\
\text { filière }\end{array}$ & $\begin{array}{l}\text { Sciences } \\
\text { naturelles } \\
\text { et mathé- } \\
\text { matiques }\end{array}$ & $\begin{array}{c}\text { Géo. } \\
\text { humaine et } \\
\text { sc. } \\
\text { humaines }\end{array}$ & $\begin{array}{c}\text { Géo. } \\
\text { physique et } \\
\text { géologie }\end{array}$ & $\begin{array}{c}\text { Géomati- } \\
\text { que et } \\
\text { informati- } \\
\text { que }\end{array}$ & $\begin{array}{c}\text { Travail de } \\
\text { fin d'études } \\
\text { (TFE) }\end{array}$ & Stage & $\begin{array}{c}\text { Option et } \\
\text { autres }\end{array}$ \\
\hline $\begin{array}{l}\text { B.Sc. } \\
\text { Humboldt } \\
\text { Berlin }\end{array}$ & $\begin{array}{c}5 \\
2,8 \%\end{array}$ & $\begin{array}{c}20 \\
11,1 \%\end{array}$ & $\begin{array}{c}70 \\
38,9 \%\end{array}$ & $\begin{array}{c}15 \\
8,3 \%\end{array}$ & $\begin{array}{c}30 \\
16,7 \%\end{array}$ & $\begin{array}{c}20 \\
11,1 \%\end{array}$ & $\begin{array}{c}20 \\
11,1 \%\end{array}$ \\
\hline $\begin{array}{l}\text { B.A. } \\
\text { Humboldt } \\
\text { Berlin }\end{array}$ & $\begin{array}{c}5 \\
2,8 \%\end{array}$ & $\begin{array}{c}70 \\
38,9 \%\end{array}$ & $\begin{array}{c}20 \\
11,1 \%\end{array}$ & $\begin{array}{c}15 \\
8,3 \%\end{array}$ & $\begin{array}{c}30 \\
16,7 \%\end{array}$ & $\begin{array}{c}20 \\
11,1 \%\end{array}$ & $\begin{array}{c}20 \\
11,1 \%\end{array}$ \\
\hline $\begin{array}{l}\text { B.Sc. Freie } \\
\text { Berlin }\end{array}$ & $\begin{array}{c}5 \\
2,8 \% \\
\end{array}$ & $\begin{array}{c}39 \\
21,7 \% \\
\end{array}$ & $\begin{array}{c}34 \\
18,9 \% \\
\end{array}$ & $\begin{array}{c}10 \\
5,6 \%\end{array}$ & $\begin{array}{c}12 \\
6,7 \%\end{array}$ & $\begin{array}{c}30 \\
16,7 \% \\
\end{array}$ & $\begin{array}{c}50 \\
27,8 \% \\
\end{array}$ \\
\hline $\begin{array}{l}\text { B.Sc. } \\
\text { Göttingen }\end{array}$ & $\begin{array}{c}6 \\
3,3 \%\end{array}$ & $\begin{array}{c}17 \\
9,4 \%\end{array}$ & $\begin{array}{c}25 \\
13,9 \%\end{array}$ & $\begin{array}{c}20 \\
11,1 \%\end{array}$ & $\begin{array}{c}12 \\
6,7 \%\end{array}$ & $\begin{array}{c}30 \\
16,7 \%\end{array}$ & $\begin{array}{c}70 \\
38,9 \%\end{array}$ \\
\hline $\begin{array}{l}\text { B.Sc. } \\
\text { Heidelberg }\end{array}$ & $\begin{array}{c}0 \\
0 \% \\
\end{array}$ & $\begin{array}{c}41,5 \\
25,8 \%\end{array}$ & $\begin{array}{c}41,5 \\
25,8 \% \\
\end{array}$ & $\begin{array}{c}34 \\
21,1 \% \\
\end{array}$ & $\begin{array}{c}22 \\
13,7 \% \\
\end{array}$ & $\begin{array}{c}0 \\
0 \%\end{array}$ & $\begin{array}{c}22 \\
13,7 \% \\
\end{array}$ \\
\hline B.Sc. Bonn & $\begin{array}{c}5 \\
3,2 \%\end{array}$ & $\begin{array}{c}56 \\
36,4 \% \\
\end{array}$ & $\begin{array}{c}57 \\
37,0 \% \\
\end{array}$ & $\begin{array}{c}10 \\
6,5 \%\end{array}$ & $\begin{array}{c}14 \\
9,1 \%\end{array}$ & $\begin{array}{c}12 \\
7,8 \%\end{array}$ & $\begin{array}{c}0 \\
0 \%\end{array}$ \\
\hline B.Sc. Cologne & $\begin{array}{c}9 \\
8,1 \%\end{array}$ & $\begin{array}{c}24 \\
21,6 \%\end{array}$ & $\begin{array}{c}24 \\
21,6 \%\end{array}$ & $\begin{array}{c}15 \\
13,5 \%\end{array}$ & $\begin{array}{c}27 \\
24,3 \%\end{array}$ & $\begin{array}{c}12 \\
10,8 \%\end{array}$ & $\begin{array}{c}0 \\
0 \%\end{array}$ \\
\hline
\end{tabular}

Source : sites internet des universités (septembre et octobre 2016)

Bien que les bacheliers en géographie organisés en Allemagne se caractérisent par un programme très fourni en géographie physique, nous n'y avons pas identifié de cours de base en physique, chimie ou biologie. Les crédits mentionnés dans la première colonne du tableau 4 ne correspondent donc qu'à des cours de statistiques. Par contre, des cours de chimie et de physique sont organisés dans les programmes de B.Sc. en géosciences, à l'image du programme organisé par l'Université d'Heidelberg. 
41 Comme pour le cas français, l'absence des sciences physiques et chimiques dans les programmes de géographie laisse supposer que les prérequis à l'étude de la climatologie ou de la géomorphologie sont inclues au sein des «unités d'enseignement » concernées. De même, notre analyse des programmes n'a pas conduit à identifier de cours connexes en sciences humaines (histoire, économie, sociologie...). Cela laisse également supposer que les savoirs relatifs à ces matières sont directement enseignés dans les cours de géographie humaine.

En comparaison des universités françaises, l'enseignement de la géomatique apparaît moins important dans les universités allemandes. Le nombre de crédits au sein de la plupart des universités analysées représente ici un ordre de grandeur de 15 crédits (à l'exception toutefois de l'Université d'Heidelberg), alors que la moyenne française est supérieure à 20 crédits. Le faible poids de la géomatique dans les formations allemandes découle vraisemblablement $\mathrm{du}$ fait que la géomatique et la géométrologie correspondent à des filières d'enseignement distinctes qui ne dépendent pas des instituts de géographie.

43 En comparaison des universités françaises, une autre différence correspond au faible poids des matières relatives à l'aménagement et, en particulier, à l'absence de spécialisations ou d'orientations qui seraient spécifiques à ce domaine. L'hypothèse explicative que nous pouvons avancer à ce propos est que la tendance vers la professionnalisation des formations de géographes s'est opérée en Allemagne dans un contexte où l'enseignement supérieur de l'aménagement était déjà bien structuré. Il s'agit là d'une situation qui rapproche le cas allemand du cas britannique, où il existe également une longue tradition de départements et de filières spécifiquement dédiés au domaine de l'aménagement (urban and regional planning).

\section{Le Royaume-Uni}

44 À l'image des universités allemandes, les universités britanniques organisent des Bachelor of Arts et des Bachelor of Science en géographie, selon l'orientation donnée aux cours. En comparaison de l'offre disponible en Allemagne, certaines institutions britanniques se distinguent par le nombre élevé de formations proposées. À titre d'exemple, l'Université de Leeds ne propose pas moins de 11 cursus géographiques différents, la plupart d'entre eux combinant cette discipline avec l'histoire, la sociologie, la géologie, les transports, l'économie ou encore le management.

La multiplicité des orientations proposées et la structuration des unités d'enseignement en large blocs de cours ne permettent pas d'établir une quantification précise des crédits en fonction des types de cours. Il apparait seulement possible de mettre en évidence l'importance des questions environnementales dans les formations et la forte hétérogénéité de leurs contenus. Concernant le Royaume-Uni, nous bénéficions toutefois d'une littérature bien développée qui permet de cerner le profil de la géographie humaine Outre-Manche.

Un premier point à souligner est que la géographie humaine britannique a une haute estime d'elle-même! En effet, sur la base d'une analyse récente ayant cherché à l'évaluer vis-à-vis des géographies humaines pratiquées à l'étranger, elle se considère tout simplement comme le leader mondial de la discipline :

In our judgement, documentary and oral evidence support the conclusion that UK

human geography is empirically and conceptually innovative, diverse, vibrant, and 
is resourcefully navigating the institutional environment of UK higher education. In many subdisciplines it is world leading, setting the intellectual agenda and providing articulate spokespersons and persuasive authors to present new knowledge and fresh conceptual insights. The field is radically interdisciplinary in its projects, partnerships, and publications; the geographical imagination seems inherently to cross boundaries. It absorbs new insights and is in a state of constant re-invention. The quality of its undergraduate students is superior to other social science disciplines according to secondary education results, and this quality moves successively up the student and faculty hierarchy. We note from bibliometric data that UK human geography surpasses in volume and citation impact the output from other countries and also exceeds comparator disciplines in the UK on most bibliometric indicators. Cumulatively, this evidence supports the conclusion that human geography as a whole in the UK ranks first in the world (ESRC-RGS-AHRC, 2013, p. 5).

Bien que se considérant comme le leader mondial de la discipline, la géographie humaine britannique souligne une faiblesse importante quant à la capacité limitée des géographes britanniques en méthodes quantitatives :

The sub-discipline faces significant challenges. Most importantly, there is a relatively small cohort of young geographers taking up these research interests given the reduction of training in quantitative methods in most undergraduate and postgraduate geography programmes, and diminished departmental priorities in this research area, most surprising in light of the growth of this field, particularly GIS, in the US. If the current trend continues, UK geographers may soon lack the skills necessary to analyse and interpret the large data sets mentioned above, although several of the methods have been developed in UK (ESRC-RGS-AHRC, 2013, p. 12).

Un autre point à souligner concernant la géographie humaine britannique est l'hétérogénéité en matière d'appartenance institutionnelle. Cet élément, qui apparaissait déjà au travers du tableau 1, est confirmé par Hall et al. (2015) qui démontrent qu'il n'existe pas de modèle dominant au Royaume-Uni. Outre-Manche, les départements de géographie semblent autant appartenir à des facultés liées aux sciences sociales qu'à des facultés scientifiques. Un deuxième élément mis en avant par les investigations de Hall et al. (2015) est que, en dépit de cette hétérogénéité, la plupart des départements continuent d'intégrer tant la composante humaine que la composante physique de la discipline, à la différence de ce qui est par exemple pratiqué aux Pays-Bas.

Une autre différence entre la géographie humaine britannique et la géographie humaine néerlandaises se rapporte aux relations avec le domaine de l'aménagement. Pour rappel, aux Pays-Bas, à l'image de la France, nous sommes en présence d'une géographie qui s'est fortement rapprochée de l'aménagement. Rien de tel n'apparaît au Royaume-Uni, où les deux types de cursus sont toujours clairement différenciés. Comme nous l'avons signalé ci-dessus, cette situation semble s'expliquer par le fait que, lorsque la géographie a engagé son tournant vers des cursus plus professionnalisant, le domaine de l'aménagement était déjà bien structuré et institué au Royaume-Uni, tant sur le plan de la profession (town planner) que sur les plans de l'enseignement universitaire et de la recherche qui l'accompagne. 


\section{La Suisse romande}

50 En Suisse, l'enseignement de la géographie est dispensé au sein de quatre universités francophones: l'Université de Genève, l'Université de Lausanne, l'Université de Fribourg et l'Université de Neufchâtel. À Genève et à Neufchâtel, la géographie appartient à des facultés de sciences humaines, ce qui s'apparente au modèle français. À Lausanne et à Fribourg, le positionnement institutionnel de la géographie se rapproche davantage du modèle allemand. À Fribourg, la géographie appartient au Département des géosciences, qui appartient lui-même à la Faculté des sciences. Dans cette université, le Département des géosciences organise deux filières de bachelier: une filière en géographie et une filière en sciences de la Terre. À Lausanne, nous trouvons une autonome Faculté des géosciences et de l'environnement. Cette faculté organise un cursus de bachelier en " géosciences et environnement » qui propose trois orientations : la géographie, la géologie et les sciences de l'environnement.

51 Le tableau 5 porte sur la ventilation des crédits délivrés dans les formations de bachelier assurées à Fribourg, Genève et Lausanne. Le cas de Neufchâtel n'est pas pris en compte car la présentation du programme disponible ne nous a pas permis une comptabilisation des crédits. Afin d'établir le tableau 5 , nous nous sommes appuyés sur la méthode utilisée pour le tableau 2 préparé pour la Belgique, en appliquant une comptabilité qui maximise le poids de la rubrique "géographie humaine et sciences humaines".

Le cas suisse témoigne de l'impact des appartenances institutionnelles sur les programmes de formation. Par exemple le programme genevois de B.A. est très fortement orienté vers les sciences humaines. En comparaison des autres programmes consultés, nous y trouvons un poids très important de disciplines connexes à la géographie humaine (sciences politiques, économie politique...). Pour Genève, nous sommes également frappés par l'absence totale des sciences naturelles et des mathématiques. Cela semble en partie résulter de l'intégration des matières de statistiques dans des modules dédiés aux méthodes quantitatives en sciences humaines.

Tableau 5. Crédits par types de cours pour les formations de bachelier en géographie au sein des universités de Fribourg, Genève et Lausanne - Calculs établis sur la base d'une maximisation des cours en géographie humaine et sciences humaines.

\begin{tabular}{|l|c|c|c|c|c|c|}
\hline Université & $\begin{array}{c}\text { Sciences } \\
\text { naturelles } \\
\text { et mathé- } \\
\text { matiques }\end{array}$ & $\begin{array}{c}\text { Géo. } \\
\text { humaine et } \\
\text { sc. } \\
\text { humaines }\end{array}$ & $\begin{array}{c}\text { Géo. } \\
\text { physique et } \\
\text { géosciences }\end{array}$ & $\begin{array}{c}\text { Géomatique } \\
\text { et } \\
\text { informatique }\end{array}$ & Autres & TOTAL \\
\hline B. Sc. & 16 & 99,5 & 47,5 & 14 & 3 & 180 \\
Fribourg & $8,9 \%$ & $55,3 \%$ & $26,4 \%$ & $7,8 \%$ & $1,7 \%$ & $100 \%$ \\
\hline B. Sc. & 18 & 105 & 33 & 18 & 6 & 180 \\
Lausanne & $10,0 \%$ & $58,3 \%$ & $18,3 \%$ & $10,0 \%$ & $3,3 \%$ & $100 \%$ \\
\hline B.A. Genève & 0 & 156 & 6 & 18 & 0 & 180 \\
\hline
\end{tabular}

Source : sites internet des universités (décembre 2016)

53 Le programme de B.Sc. dispensé à Fribourg est davantage orienté vers les sciences, avec un poids marqué pour les enseignements du domaine de la géographie physique. Cette situation s'explique par l'appartenance de la géographie fribourgeoise à la faculté des sciences. Pour autant, en comparaison de la situation belge, les étudiants qui souhaitent maximiser leur programme en géographie humaine et sciences humaines y trouvent un 
terrain beaucoup plus favorable (part maximale de 55,3\% contre 21,7 \% à l'ULiège par exemple). La situation à Lausanne se caractérise par une configuration intermédiaire entre les situations fribourgeoise et genevoise.

À l'instar des autres formations analysées en dehors du cadre belge, le cas suisse se caractérise également par l'absence de formations de base en sciences physique, chimique ou biologique. L'analyse des programmes montre toutefois que de tels cours sont proposés aux étudiants lausannois qui choisissent les orientations géologie et sciences de l'environnement du bachelier « géosciences et environnement ».

\section{Discussion des résultats et conclusions}

\section{Les contextes institutionnels et les programmes de cours}

\section{Des contextes institutionnels hétérogènes}

Une première conclusion qui se dégage de nos investigations correspond aux grandes hétérogénéités des contextes institutionnels au sein desquels s'inscrivent les domaines universitaires de la géographie en général et de la géographie humaine en particulier. Malgré la convergence des systèmes d'enseignements supérieurs et malgré l'internationalisation croissante de la recherche, la diversité des configurations institutionnelles est la norme. Ce constat tient au fait que la géographie appartient à la fois aux sciences naturelles et aux sciences humaines, voire également aux sciences de l'ingénieur par sa composante géomatique. Un tel profil ouvre évidemment la porte à une multiplicité d'organisations institutionnelles puisqu'il ne correspond pas au découpage classique des disciplines scientifiques sur lequel s'est calquée la structure de la plupart des universités. Plusieurs universités ont résolu cette tension en innovant institutionnellement pour mettre en place des facultés ou des structures équivalentes consacrées à l'environnement et aux sciences géographiques.

\section{Le poids exceptionnel des matières connexes en Belgique}

Une conclusion plus surprenante correspond au différentiel entre les programmes belges et étrangers concernant le poids des matières connexes à la géographie. En effet, les programmes étrangers font clairement moins de place à ces matières connexes, qu'elles appartiennent aux sciences humaines ou aux sciences naturelles et exactes. $\mathrm{Ce}$ décalage semble témoigner de philosophies distinctes quant aux relations entre la géographie et ces autres disciplines. En Belgique, nous trouvons une conception qui tend à considérer certaines sciences connexes comme des sciences de base. Par exemple, il y est généralement considéré qu'il faut maîtriser les bases de la physique avant de se lancer dans la géographie physique ou les bases de l'économie avant de se lancer dans la géographie économique. À l'étranger, une telle conception semble étrangère à la philosophie des programmes. Cette observation est importante notamment vis-à-vis de la problématique des prérequis. En effet, nous pouvons en déduire que ce sont les enseignants en charge des cours de géographie qui, au sein des universités étrangères, assurent l'essentiel des mises à niveau en sciences connexes. 


\section{L'impact des contextes institutionnels sur la variété des programmes} la matière, nous pouvons souligner le rôle déterminant des jeux d'acteurs dans l'articulation entre ces programmes et les contextes institutionnels. Illustrons cette idée par le constat du poids important des disciplines scientifiques dans les programmes des départements de géographie qui appartiennent à des facultés des sciences ou des géosciences. formation, invoquons également le contenu de l'offre des universités de Liège (ULiège) et de Gand (UGent). En effet, suite à l'influence du développement d'un master en géomatique-géométrologie, les programmes de bachelier offerts par ces institutions intègrent un volume de cours appartenant au domaine de la géomatique plus conséquent qu'au sein des autres universités, qu'elles soient belges ou étrangères.

\section{Trois modèles distincts}

Il ressort de notre recherche qu'il est opportun de différencier trois modèles distincts quant au positionnement des domaines universitaires de la géographie et de la géographie humaine.

\section{Le modèle " géographie diversifiée et autonome par rapport à l'aménagement »}

60 Ce modèle caractérise notamment l'Allemagne et le Royaume-Uni, où nous trouvons des formations diversifiées qui appartiennent tantôt au domaine des sciences (B.Sc.), tantôt au domaine des sciences humaines (B.A.). Ce modèle permet aux étudiants de trouver des formations adaptées à leurs préférences dès leur entrée à l'université. En parallèle, ce modèle offre des opportunités de développement et d'épanouissement tant pour les aspects humains de la géographie que pour ses aspects « science naturelle».

61 La Suisse romande s'inscrit également dans la configuration d'une offre diversifiée. Malgré l'offre limitée induite par la taille limitée de la population suisse francophone, des programmes fortement différenciés y coexistent. D'un côté, le B.A. de l'Université de Genève est un programme quasi exclusivement constitué de cours relevant des sciences humaines. D'un autre côté, à l'autre bout du spectre, le B.Sc. de l'Université de Fribourg propose une offre qui se rapproche des B.Sc. allemands.

Qu'il s'agisse de l'Allemagne ou du Royaume-Uni, nous avons également trouvé une offre de formation qui intègre finalement assez peu les matières liées au domaine de l'aménagement des territoires. Cette configuration d'une géographie autonome vis-àvis de l'aménagement s'oppose aux situations de la France et des Pays-Bas, deux pays qui appartiennent plutôt au modèle "géographie-aménagement ".

\section{Le modèle « géographie-aménagement »}

63 Le modèle "géographie-aménagement" repose sur une proximité forte entre la géographie humaine et l'aménagement des territoires. L'existence de ce modèle tient à l'enjeu de l'employabilité avec, lors des dernières décennies, la recherche de la diversification des débouchés par le développement de cursus orientés vers les pratiques de l'aménagement et de l'urbanisme. Comme nous l'avons détaillé ci-dessus, 
ni l'Allemagne, ni le Royaume-Uni n'ont connu une telle évolution et la séparation y demeure nette entre la géographie humaine, d'une part, et l'aménagement des territoires, d'autre part. Comme nous l'avons également détaillé ci-dessus, la situation d'une géographie autonome qui caractérise tant l'Allemagne que le Royaume-Uni semble s'expliquer par l'institutionnalisation ancienne du domaine académique de l'aménagement et, dès lors, par une occupation précoce de ce terrain par des diplômes spécifiques.

Précisons que le rapprochement entre la géographie humaine et l'aménagement des territoires qui caractérise le modèle "géographie-aménagement » ne correspond en rien à une fusion des deux domaines. En effet, qu'il s'agisse de la France ou des Pays-Bas, les départements de géographie ne disposent pas du monopole sur les formations en urbanisme et aménagement, dont certaines sont également organisées par des écoles d'ingénieurs ou d'architecture. Les formations assurées par les ingénieurs et les architectes apparaissent complémentaires des formations assurées par les géographes. Elles sont souvent plus techniques et davantage orientées vers les échelles fines de l'urbanisme (urban design) plutôt que vers les échelles plus larges de la ville et de la région (urban and regional planning).

Concernant les situations en France et aux Pays-Bas, précisons encore que les recherches des géographes humains sont loin de se limiter aux problématiques de l'aménagement des territoires. En réalité, la plupart des chercheurs français et néerlandais qui sont actifs en géographie humaine continuent de traiter de sujets qui s'inscrivent en dehors de ces problématiques. Cette observation est à relier au fait que les universités françaises et néerlandaises intègrent des programmes de master qui offrent tant des parcours "géographie au sens strict » que des parcours "géographieaménagement » considérés comme plus professionnalisants.

\section{Le modèle " géographie par les sciences de base »}

En Belgique, les formations universitaires en géographie n'appartiennent pas aux deux modèles précédents. La situation belge se différencie clairement du modèle " géographie diversifiée et autonome" car le poids des matières scientifiques y est bien plus considérable qu'au sein des programmes les plus scientifiques offerts en Allemagne ou au Royaume-Uni. Nous trouvons donc en Belgique un modèle différent, dont la spécificité est notamment liée à une conception qui fait de certaines sciences connexes des sciences de base.

67 En matière d'enseignement, le modèle belge "géographie par les sciences de base " se caractérise par un poids relativement important de la géographie physique et de la géomatique mais, surtout, par un poids hors norme des sciences naturelles et exactes (la physique et la chimie en particulier). Nous parlons d'un poids hors norme car ces enseignements en sciences de base sont absents des programmes de géographie proposés à l'étranger. Au sein des universités étrangères prises en compte par nos investigations, des cours de physique et de chimie ont été identifiés dans des programmes de géosciences, de géologie ou de sciences de l'environnement, mais jamais dans des programmes de géographie.

68 La spécificité du cas belge conduit à souligner la question des prérequis. En Belgique, l'enseignement de la géographie universitaire repose sur le postulat que des prérequis en physique et en chimie sont nécessaires pour les enseignements de géographie 
physique. Étonnamment, seul le système belge est structuré sur la base d'un tel postulat. Il semble donc que les prérequis enseignés dans des unités d'enseignement distinctes en Belgique soient enseignées à l'étranger au sein d'unités d'enseignement davantage intégrées associant structurellement les prérequis et leurs finalités géographiques. Ce décalage n'est pas neutre sur le plan pédagogique et il devrait être étudié plus en détail.

Dans un contexte structurel de faible recrutement des étudiants, nous pouvons nous interroger sur les raisons qui ont permis la perpétuation du modèle « géographie par les sciences de base ». Une partie de la réponse se trouve vraisemblablement dans l'inertie par rapport au modèle général de l'universitaire qui doit disposer de connaissances étendues et au modèle particulier du géographe qui doit disposer de connaissances étendues en sciences naturelles. Une autre partie de la réponse se trouve vraisemblablement dans l'idée selon laquelle une formation scientifique solide contribue à améliorer la qualité des cursus et la performance des diplômés. Cette hypothèse n'a, à notre connaissance, jamais été testée et mériterait d'être validée ou invalidée par une analyse objective.

En raison de son orientation vers les sciences naturelles et exactes, le modèle belge de la « géographie par les sciences de base » marginalise la géographie humaine. Dans le cadre restreint des trois années de bachelier, ce domaine ne peut s'y positionner que de manière minoritaire. Cette configuration a au moins deux conséquences directes. Premièrement, la faible marge de manœuvre au sein des programmes entraîne une relative saturation de ceux-ci au détriment d'innovations thématiques ou pédagogiques. Deuxièmement, elle éloigne de la filière géographique des étudiants intéressés par les dimensions humaines des enjeux environnementaux et territoriaux. Or, dans un même temps, les métiers liés à l'environnement et aux territoires sont de plus en plus occupées par des diplômés de disciplines connexes.

\section{BIBLIOGRAPHIE}

ARROWSMITH C., BAGOLY-SIMÓ P., FINCHUM A., ODA K. \& PAWSON E. (2007), « Student employability and its implications for geography curricula and learning practices ", Journal of Geography in Higher Education, 35, 3, pp. 365-377.

BAILLY A. (dir.) (1995), « Introduction », Les Concepts de la Géographie Humaine, Masson, Paris, pp. $11-22$.

BREUER C. (2009), « La géographie, ça sert, d'abord, à faire le Monde », Bulletin de la Société géographique de Liège, 52, pp. 49-52.

CAILLEUX A. (1973), « Sur le schéma de la géographie de P. Dagenais », Cahiers de Géographie du Québec, 42, pp. 489-492.

DÉPARTEMENT DE GÉOGRAPHIE DE L'UNIVERSITÉ DE LIÈGE (2015), Rapport d'auto-évaluation de la Commission chargée de l'évaluation AEQUES du cursus en sciences géographiques. 
DÉZERT B., BASTIÉ J. (2004), « La géographie en France », Belgeo, 1, pp. 69-82.

ESRC-RGS-AHRC (2013), International Benchmarking Review of UK Human Geography.

GREGORY D. (2009), in GREGORY D., JOHNSTON R., PRATT G., WATTS M.J. \& WHATMORE S. (eds.), The dictionary of Human Geography. $5^{\text {th }}$ Edition, Malden, Wiley-Blackwell, pp. 287-295.

HALL T., TOMS P., MCGUINNESS M., PARKER C. \& ROBERTS N. (2015), « Where's the Geography department? The changing administrative place of Geography in UK higher education ", AREA, 47, 1, pp. 56-64.

LENG S., ZHANG W., HE S., HE C., XUE D., YUAN L. \& TANG Q. (2017), « General Trends in the Geographical Sciences », Geographical Sciences During 1986-2015, Springer Singapore, pp. 3-15.

MURPHY A.B. (2007), « Geography's place in higher education in the United States », Journal of Geography in Higher Education, 31, 1, pp. 121-141.

VAN DER VAART R., DE PATER B. \& OOST K. (2004), « Geography in the Netherlands », Belgeo, 1, pp. 135-144.

VILES H. (2005), « A divided discipline? », in CASTRÉE N., ROGERS A. \& SHERMAN D. (eds.), Questioning Geography: Fundamental Debates, Malden, Wiley-Blackwell, pp. 26-38.

WHALLEY W.B., SAUNDERS A., LEWIS R.A., BUENEMANN M. \& SUTTON P.C. (2011), « Curriculum development: Producing geographers for the $21^{\text {st }}$ century », Journal of Geography in Higher Education, 35, 3, pp. 379-393.

\section{NOTES}

1. Cette liste, bien que non datée, semble correspondre à une situation relativement récente. Elle

a été consultée à partir de l'adresse suivante: http://infogeo.univ-paris1.fr/Fichiers/ Universites_enseignants.htm.

2. À titre d'illustration, une enquête menée en 2015 par l'Association des alumni en géographie de l'Université de Liège auprès de 165 répondants diplômés de l'ULiège entre 1956 et 2014 donne les débouchés suivants : aménagement du territoire, développement des territoires urbains et ruraux, urbanisme ( $26 \%$, en croissance) ; géométrologie, géomatique, cartographie, télédétection ( $24 \%$, en croissance) ; pédagogie et didactique ( $22 \%$, en baisse) ; environnement ( $7 \%$, stable) ; climatologie et météorologie (7\%, en croissance); géomorphologie et gestion des risques naturels ( $3 \%$, stable); autres ( $11 \%$, en baisse).

3. European Credit Transfer and Accumulation System, chaque crédit correspondant à une unité temporelle de travail pour l'étudiant. Une année complète comporte 60 ECTS.

4. Les orientations « mineures » correspondent à une partie minoritaire du cursus des étudiants au sein de laquelle sont regroupés des cours cohérents sur le plan disciplinaire. Pour la géographie, ce système existe à l'UCL, où le bachelier en géographie intègre une majeure en 90 crédits et trois possibilités de mineures en 30 crédits : une mineure d'approfondissement en géographie, une mineure en économie et une mineure en physique. En parallèle, les autres programmes de bachelier de la faculté des sciences de l'UCL offrent la possibilité d'une mineure de 30 crédits en géographie.

5. Les programmes des universités suivantes ont été consultés: l'Université de Lille 1, l'Université Grenoble-Alpes, l'Université d'Angers, l'Université Paris 1 Panthéon-Sorbonne et l'Université de Lorraine. 


\section{RÉSUMÉS}

La recherche synthétisée dans cet article porte sur l'enseignement universitaire de la géographie humaine. Elle est basée sur une comparaison des programmes de bachelier en géographie au sein de six pays et elle a permis de mettre en exergue la coexistence de trois modèles distincts: l'enseignement d'une "géographie-aménagement » en France et aux Pays-Bas ; l'enseignement d'une " géographie diversifiée et autonome par rapport à l'aménagement » au Royaume-Uni, en Allemagne et en Suisse; l'enseignement d'une "géographie par les sciences de base " en Belgique. $\mathrm{Vu}$ le poids considérable des sciences naturelles de base dans ce dernier modèle, la Belgique occupe ici une position originale qui n'apparaît guère favorable à l'épanouissement de la géographie humaine. Nous en concluons qu'il est opportun de porter la réflexion sur l'évolution du modèle en question.

The research synthesized in this article focuses on university education of human geography. It is based on a comparison of the programs of Bachelor in geography in six countries and it helped to highlight the co-existence of three distinct models: the teaching of a "geography and planning" in France and the Netherlands; the teaching of a "diverse and autonomous geography against planning" in the UK, Germany and Switzerland; the teaching of a "geography by the basic sciences" in Belgium. Given the considerable weight of basic natural sciences in the latter model, Belgium occupies an original position that is not favorable to the growth of human geography. We therefore conclude that it is opportune to reflect on the evolution of the model in question.

\section{INDEX}

Keywords : human geography, spatial planning, geography education, natural sciences, epistemology, Belgian Universities, European Universities

Mots-clés : géographie humaine, aménagement du territoire, enseignement de la géographie, sciences naturelles, épistémologie, universités belges, universités européennes

\section{AUTEURS}

JEAN-MARIE HALLEUX

Université de Liège, ECOGEO et LEPUR, Jean-Marie.Halleux@uliege.be

\section{CHRISTOPHE BREUER}

Université de Liège, ECOGEO et LEPUR, Christophe.Breuer@uliege.be 